\title{
THE SPACE OF HENSTOCK INTEGRABLE FUNCTIONS OF TWO VARIABLES
}

\section{KRZYSZTOF OSTASZEWSKI}

\author{
University of Louisville \\ Department of Mathematics \\ Louisville, KY 40292 \\ U.S.A.
}

(Received February 6, 1987 and in revised form March 15, 1987)

ABSTRACT. We consider the space of Henstock integrable functions of two variables. Equipped with the Alexiewicz norm the space is proved to be barrelled. We give a partial description of its dual. We show by an example that the dual can't be described in a manner analogous to the one-dimensional case, since in two variables there exist functions whose distributional partials are measures and which are not multipliers for Henstock integrable functions.

KEY WORDS AND PHRASES: Henstock integra1, barre1, barrelled space, normed space, continuous linear functionals.

1980 MATHEMATICS SUBJECT CLASSIFICATION. PRIMARY 46E10. SECONDARY 26 A39.

1. DEFINITION.

We will write $I_{0}=[0,1]^{2}$. A function $f: I_{0} \rightarrow R$ is Henstock integrable, with

$$
\int_{I_{0}} f(x, y) d x d y
$$

written for the value of the integral, if for every $\varepsilon>0$ there exists a positive $\delta: I_{0} \rightarrow R$ such that if

$$
\pi=\left\{\left(\left(\mathrm{x}_{i}, \mathrm{y}_{\mathbf{i}}\right), \mathrm{I}_{\mathbf{i}}\right): i=1,2, \ldots, \mathrm{n}\right\}
$$

is a partition of $\mathrm{I}_{0}$ (i.e., $\mathrm{I}_{i}$ 's are nonoverlapping subintervals of $\mathrm{I}_{0}$ whose union is $I_{0}$ ) for which

$$
\left(\mathrm{x}_{i}, \mathrm{y}_{i}\right) \varepsilon \mathrm{I}_{i} \subset \Delta\left(\left(\mathrm{x}_{i}, \mathrm{y}_{i}\right), \delta\left(\mathrm{x}_{i}, \mathrm{y}_{i}\right)\right) \text {, }
$$

where $\Delta((a, b), r)$ stands for the disk centered at $(a, b)$ of radius $r$, then

$$
\left|\sum_{i=1}^{n} f\left(x_{i}, y_{i}\right) \lambda\left(I_{i}\right)-\int_{I_{0}} f(x, y) d x d y\right|<\varepsilon,
$$

where $\lambda\left(I_{i}\right)$ denotes the area of $I_{i}$.

We will write $H$ for the class of Henstock integrable functions on $I_{0} \cdot H$ is a linear space. If we replace $\lambda\left(I_{i}\right)$, for $I_{i}=\left[a_{i}, b_{i}\right] x\left[c_{i}, d_{i}\right]$, in (4) by $g\left(a_{i}, c_{i}\right)-g\left(a_{i}, d_{i}\right)-g\left(b_{i}, c_{i}\right)+g\left(b_{i}, d_{i}\right)$, for a certain $g: I_{0} \rightarrow \mathbb{R}$, then we obtain the definition of the Henstock integral of $f$ with respect to $g$, written as $\int_{I_{0}} \mathrm{Idg}$.

Henstock integral in the plane is fully discussed in [7]. 
2. DEFINITION.

Let $\mathrm{f} \varepsilon H$, set

$$
f(x, y)=\int_{[0, x] \times[0, y]} f(s, t) d s d t .
$$

It is shown in [3] (page 549) that $f$ is continuous. Let

$$
|| f||=\sup _{(x, y) \in I_{0}}|f(x, y)| .
$$

We will call (6) the Alexiewicz norm on $H$.

3. PROPOSITION.

$\mathrm{T} \varepsilon H^{*}$ if and only if there is a finite signed Borel measure $\mu$ on $(0,1] \times(0,1]$ such that

$$
T(f)=\int_{I_{0}} F(x, y) d \mu(x, y)
$$

The norm of $T$ is equal to the norm of $\mu$.

PROOF. Let $C$ be the space of continuous real-valued functions on $I_{0}$. Define

$$
C_{0}=\{F \in C: F(x, y)=0 \text { if } x=0 \text { or } y=0\}
$$

Then if we assign

$$
H 3 \mathrm{f} \rightarrow \tilde{\mathrm{f}} \varepsilon \mathrm{C}_{0}
$$

$H$ is mapped isomorphically into a dense subset of $\mathrm{C}_{0}$ (since every polynomial is the indefinite Henstock integral of its second mixed partial). Thus, we can 1dentify H* with $\mathrm{C}_{0} *$. But $\mathrm{C}_{0}$ is a closed subspace of $\mathrm{C}$ and $\mathrm{C}_{0} *=\mathrm{C} * / \mathrm{C}_{0}^{1}$, which may be seen to be the space of finite signed Borel measures on $(0,1] \times(0,1]$. (7) follows from the general form of a continuous linear functional on $\mathrm{C}_{0}$.

4. DEFINITION. $\mathrm{fg} \varepsilon H$.

A function $g: I_{0} \rightarrow \mathbb{R}$ is a multiplier for $H$ if for every $f \varepsilon H$ we have also

\section{REMARK.}

In the one-dimensional case the dual of the space of Henstock-integrable functions is given by the class of multipliers (see [6]). The multipliers are functions whose distributional derivatives are measures. The two-dimensional case is different.

In [4] Kurzweil defines $g: I_{0} \rightarrow \mathbb{R}$ to be of strongly bounded variation if for every $x, g(x, \cdot)$ is of bounded variation, for every $y, g(\cdot, y)$ is of bounded variation, and

$$
M(g)=\sup \sum_{i=1}^{n}\left|g\left(a_{i}, c_{i}\right)-g\left(a_{i}, d_{i}\right)-g\left(b_{i}, c_{i}\right)+g\left(b_{i}, d_{i}\right)\right|<+\infty
$$

where sup is taken over all partitions lof $\left.I_{0}\right)\left\{I_{i}\right\}_{i=1}^{n}, I_{i}=\left[a_{i}, b_{i}\right] \times\left[c_{i}, d_{i}\right]$, consisting of non-overlapping, nondegenerate closed intervals. Then he shows that functions of strongly bounded vartation are multipliers for $H$, and for $f \in H, g$ of strong1y bounded variation.

$$
\left|\int_{I_{0}} \tilde{f}(x, y) d g(x, y)\right| \leq\|f\| M(g),
$$

so that every $g$ of strongly bounded variation is a continuous linear functional on H. 
The connection between this result and Proposition 3 is not known. It is not known either if functions of strongly bounded variation and those equivalent to them are the only multipliers.

6. EXAMPLE.

There exists a function $g: I_{0} \rightarrow R$ whose distributional partials are measures and which is not a multiplier. Define

$$
g(x, y)= \begin{cases}\sqrt[6]{x-y} & \text { for } x \geq y \\ 0 & \text { otherwise }\end{cases}
$$

Note that Krickeberg shows in [2] that $\mathrm{g}: \mathrm{I}_{0} \rightarrow \mathrm{R}$ has its distributional partials being measures if and only if it is of bounded variation in the sense of Tonel1i.

$$
\begin{array}{r}
\text { For } g, \operatorname{var} g(\cdot, y)=\sqrt[6]{1-y}, \operatorname{var} g(x, \cdot)=\sqrt[6]{x} \text { and } \\
\int_{0}^{1} \sqrt[6]{1-y} d y+\int_{0}^{1} \sqrt[6]{x} d x \leq 2 .
\end{array}
$$

So $g$ is of bounded variation in the sense of Tone11i.

$$
\begin{aligned}
& \text { Define for } \begin{aligned}
& \mathrm{n} \geq 2 \\
& \mathrm{~K}_{\mathrm{n}}=\left[1-\frac{1}{\mathrm{n}-1}, 1-\frac{1}{\mathrm{n}}\right]^{2}, \\
& L_{\mathrm{n}}=\left\{(\mathrm{x}, \mathrm{y}) \varepsilon \mathrm{K}_{\mathrm{n}}: \mathrm{y} \leq \mathrm{x}\right\}
\end{aligned}
\end{aligned}
$$

and for every $n \geq 2$ construct a continuous $f_{n}: K_{n} \rightarrow R$ such that $f_{n}(x, y)=-f_{n}(y, x)$, $f_{n}$ is equal to 0 on the boundary of $K_{n}$, nonnegative on $L_{n}$ and

$$
\int_{L_{n}} f_{n}(x, y) d x d y=\frac{1}{\sqrt{n}},
$$

and $f_{n}(x, y)=0$ for every $(x, y) \varepsilon K_{n}$ such that $|x-y|<n^{-3}$. Then for $f$ given by

we have $\mathrm{f} \varepsilon H$, yet $\mathrm{fg} \notin H$.

$$
f(x, y)= \begin{cases}f_{n}(x, y) & \text { for }(x, y) \varepsilon K_{n} \text { for some } n \geq 2, \\ 0 & \text { otherwise. }\end{cases}
$$

7. REMARK.

It is shown in [8] that the space of Henstock integrable function of one variable is barrelled. We will show it to be true also in two dimensions.

8. DEFINITION.

If $E$ is a topological vector space then a set $B \subset E$ is a barrel if $B$ is closed, convex, circled and radial at 0 . A locally convex space in which every barrel is a neighborhood of 0 is termed a barrelled space. It should be noted that each barrel in a space $E$ which is of the second category in itself is necessarily a neighborhood of 0 . In particular, every Banach space is barrelled. The importance of barrelled spaces lies in the following Barrel Theorem.

9. THEOREM.

Let $E$ be a barrelled space and $F$ be a pointwise bounded family of continuous linear functions on $E$ into a locally convex space $K$. Then the family $F$ is equicontinuous. Consequent1y, in this case, $F$ is uniformly bounded on each bounded subset of $E$.

PROOF. See [5] (page 104).

This theorem implies in particular that the Banach-Steinhaus Theorem holds for barrelled spaces. 
10. DEFINITION.

Let $S$ stand for the space of real-valued additive functions $F$ of interval $I=[a, b] \times[c, d] \subset I_{0}$ for which there is a continuous $f: I_{0} \rightarrow \mathbb{R}$ such that

$$
F(I)=f(a, c)-f(a, d)-f(b, c)+f(b, d)
$$

Notice that if $F \varepsilon S$ then there is a unique $f \varepsilon C$, such that $f(x, y)=0$ if $x=0$ or $\mathrm{y}=0$, i.e., $\mathrm{f} \varepsilon \mathrm{C}_{0}$, defining it. Let

$$
|| F||=\sup _{(x, y) \varepsilon I_{0}}|f(x, y)|
$$

where $F \in S$, and $f \in C_{0}$ defines it. $S$ is a Banach space isometric to $C_{0}$.

11. THEOREM.

Let $X$ be a subspace of $S$ satisfying the following two conditions:

If $F \in X$ and $J \subset I_{0}$, and

$$
F_{J}(I)=F(I \cap J)
$$

for $I \subset I_{0}$ then $F_{J} \in X$;

(b) If $c \in I_{0}, F \in S$, and $F_{J} \varepsilon X$ for every $J \subset I_{0}$ such that if $\ell_{1}, l_{2}$ are the vertical and the horizontal line segments through $\mathrm{c}$ then $\mathrm{J} \cap l_{1}=\phi, \mathrm{J} \cap \ell_{2}=\phi$, then $F \in X$.

Then $X$ is barrelled.

PROOF. In the proof we will denote, for $z_{1}, z_{2} \varepsilon R^{2}$, by $\left[z_{1}, z_{2}\right]$ an interval for which $z_{1}, z_{2}$ are opposite vertices. Let $B$ be a barrel in $X$. If $B$ is not a neighborhood of zero, then it is nowhere dense. To show that, suppose that a barrel $B$ is not nowhere dense. There is an open set $U$ such that $U \subset B$. Since $B$ is convex and circled

$$
\frac{1}{2}(U-U) \subset \frac{1}{2}(B-B)=\frac{1}{2}(B+B) \subset B .
$$

$U$ - $U$ is a neighborhood of zero, and so is $B$.

For every $I \subset I_{0}$ write

$$
X(I)=\left\{F_{I}: F \quad \varepsilon \quad X\right\}
$$

and

$$
B(I)=B \cap X(I) \text {. }
$$

Then $B(I)$ is a barre1 in $X(I)$.

Suppose $I=I_{1} \cup \ldots . \cup I_{n}$, where $I_{1}, \ldots, I_{n}$ are nonoverlapping. Then $B\left(I_{i}\right) \subset B(I)$ for $i=1, \ldots, n$, so if $F_{i} \varepsilon B\left(I_{i}\right), i=1, \ldots, n$, then $F_{i} \varepsilon B(I)$, and, since $B(I)$ is convex,

$$
\frac{1}{n}\left(F_{1}+\ldots+F_{n}\right) \in B(I)
$$

Consequent1y, $B\left(I_{1}\right)+\ldots+B\left(I_{n}\right) \subset n B(I)$. The space $X(I)$ is a topological direct sum of $X\left(I_{1}\right), \ldots, X\left(I_{n}\right)$. If $B\left(I_{1}\right), \ldots, B\left(I_{n}\right)$ are neighborhoods of zero in $X\left(I_{1}\right), \ldots, X\left(I_{n}\right)$ (respectively) then $B(I)$ is a neighborhood of zero in $X(I)$. Thus, if $B(I)$ is nowhere dense in $X(I)$ then at least one of $B\left(I_{i}\right)^{\prime} s, i=1, \ldots, n$, is nowhere dense in the corresponding $X\left(I_{i}\right)$.

Therefore, if we divide $I_{0}$ into four subintervals by splitting the sides into halves, among so obtained intervals there is at least one, call it $I_{1}$, such that $B\left(I_{1}\right)$ is nowhere dense in $X\left(I_{1}\right)$. Applying the same procedure to $I_{1}$, and then continuing it, we obtain a sequence of intervals $I_{n}$ such that 
where $c$ is a certain point in $I_{0}$, and $B\left(I_{n}\right)$ is nowhere dense in $X\left(I_{n}\right)$ for every $\mathrm{n} \in \mathrm{N}$.

For every $\mathrm{n} \varepsilon \mathrm{N}$, write

$$
I_{n}=I_{n}^{1} \cup I_{n}^{2} \cup I_{n}^{3} \cup I_{n}^{4}
$$

where $I_{n}^{i}, i=1,2, \ldots, 4$ are subintervals of $I_{n}$ obtained from $i t$ by drawing lines parallel to its sides and going through $c$. We can assume that $I_{n}^{i}$ 's are numbered so that

$$
\mathrm{I}_{\mathrm{n}+1}^{\mathrm{i}} \subset \mathrm{I}_{\mathrm{n}}^{\mathrm{i}}
$$

for every $n$ and $i$. Notice that since $B\left(I_{n}\right)$ is nowhere dense in $X\left(I_{n}\right)$ for every $n$, there is at least one $i$ such that $B\left(I_{n}^{i}\right)$ is nowhere dense in $X\left(I_{n}^{i}\right)$.

Consider the four sequences $\left\{\mathrm{I}_{\mathrm{n}}^{i}\right\}_{n \in N}$, for $i=1,2,3,4$. If in each of them there is only finitely many $n \varepsilon N$ such that $B\left(I_{n}^{i}\right)$ is nowhere dense in $X\left(I_{n}^{i}\right)$ then after passing those finitely many indices we will get all four $B\left(I_{n}^{1}\right), i=1,2,3,4$, being neighborhoods of zero. This will force $B\left(I_{n}\right)$ to be a neighborhood of zero, a contradiction. Therefore, among the four sequences $\left\{I_{n}^{i}\right\}_{n \in N}$ there has to be one which produces infinitely many $B\left(I_{n}^{i}\right)$ 's which are nowhere dense in the corresponding $X\left(I_{n}^{i}\right)$ 's.

Let $\left\{I_{n}^{i}\right\}_{n} \varepsilon N$ be that sequence, and let $\left\{I_{n_{k}}^{i}\right\}_{k \in N}$ be its subsequence such that $B\left(I_{n_{k}}^{i_{0}}\right)$ is nowhere dense in $X\left(I_{n}^{i}{ }_{k}\right)$ for every $k \varepsilon N$. Write $J_{k}=I_{n_{k}}^{i}$ for $k \varepsilon N$, and let $\mathrm{J}_{\mathrm{k}}=\left[\mathrm{c}, \mathrm{x}_{\mathrm{k}}\right]$.

Let $u_{1}=x_{1}$. There exists a function $G_{1} \in X\left(J_{1}\right)$ such that $G_{1} \notin B$ and $\| G_{1}||<1 / 2$. Then since $B$ is closed and $\lim _{x \rightarrow c} G_{\left[x, u_{1}\right]}=G_{1}$ (in $X$ ) there is a

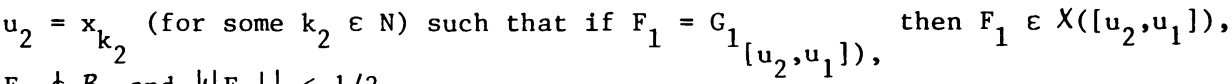
$\mathrm{F}_{1} \notin B$, and || $\mathrm{F}_{1}||<1 / 2$.

Proceeding by induction, if $n \in N$, then we have a function $G_{n} \varepsilon X\left(J_{k_{n}}\right)$ such that $G_{n} \notin n B$ and $\left\|G_{n}\right\|<1 / 2^{n}$. Since is closed and

$$
\lim _{x \rightarrow c} G_{n_{\left[x, u_{n}\right]}}=G_{n}(\text { in } X)
$$

there is a $u_{n+1}=x_{k_{n+1}}$ (for some $k_{n+1} \varepsilon N$ ) such that if $F_{n}=G_{\left.n_{\left[u_{n+1}\right.}, u_{n}\right]}$ then $\mathrm{F}_{\mathrm{n}} \varepsilon X\left(\left[\mathrm{u}_{\mathrm{n}+1}, \mathrm{u}_{\mathrm{n}}\right], \mathrm{F}_{\mathrm{n}} \in \mathrm{n} B\right.$, and || $\mathrm{F}_{\mathrm{n}}||<1 / 2^{\mathrm{n}}$.

Consider the set $A$ defined as the closed convex hull of the sequence $\left\{F_{n}\right\}$

in S. Every element of $A$ is of the form

$$
\mathrm{F}=\sum_{\mathrm{n}=1}^{+\infty} \lambda_{\mathrm{n}} \mathrm{F}
$$

for some sequence of scalars $\left\{\lambda_{n}\right\}$ with $\sum_{n=1}^{+\infty}\left|\lambda_{n}\right| \leq 1$. Take a $u \varepsilon\left[c_{1}, u_{1}\right], u \neq c$, $u \neq u_{1}$, and notice that

$$
F_{\left[u, u_{1}\right]}=\sum_{n=1}^{+\infty} \lambda_{n} F_{n_{\left[u, u_{1}\right]}}
$$


Now only finitely many terms on the right-hand side of (28) are nonzero. Therefore for every such $u, F_{\left[u, u_{1}\right]} \varepsilon X\left(\left[u, u_{1}\right]\right)$. Consequent $1 y$, by the condition $(b), A \subset X$. Therefore $B$ absorbs $A^{1}$ ( $B$ is a barrel). This, however, is a contradiction, since $B$ does not even absorb the sequence $\left\{F_{n}\right\}$. The proof is ended.

12. REMARK.

It is we11 known, and shown in [3], that

$$
\tilde{H}=\left\{\tilde{f}: f \in H\left(I_{0}\right)\right\}
$$

equipped with the Alexiewicz norm is a subspace of $\mathrm{S}$ satisfying the conditions (a), (b) of theorem 9 .

13. COROLLARY .

$H$ is barrelled.

14. COROLLARY.

If $T$ is a pointwise bounded family of continuous linear functionals on $H$ then $T$ is equicontinuous, and consequent $1 y$, uniformly bounded on each bounded subset of $H$.

15. COROLLARY.

If $\left\{g_{n}\right\}$ is a sequence of functions of strongly bounded variation on $I_{0}$ such that for every $f \in H$

exists, then

$$
\lim _{n \rightarrow \infty} \int_{I_{0}} \tilde{f}(x, y) \operatorname{dg}_{n}(x, y)
$$

$$
T(f)=\lim _{n \rightarrow \infty} \int_{I_{0}} \tilde{f}(x, y) d g_{n}(x, y)
$$

is a continuous linear functional on $H$.

We were not able to prove or disprove whether the functional (31) is itself generated by a certain function of strongly bounded variation. We do not know either whether all functionals on $H$ are of the form (31).

16. REMARK.

[8] presents a Henstock-type integral in the plane for which the classical divergence theorem holds. The integral introduced by Pfeffer integrates divergence of every differentiable vector field (unlike the Lebesgue integral).

Applying the proposition 4.10 of [8], one can show that the integral of Pfeffer satisfies the conditions (a), (b) of Theorem 11. Indefinite integral is also continuous. Thus, the space of Pfeffer-integrable functions, equipped with the Alexiewicz norm, is also barrelled.

\section{REFERENCES}

1. Henstock, R. Theory of Integration, Butterworths, London, 1963.

2. Krickeberg, K. Distributionen, Funktionen beschrankter Variation and Lebesguescher inhalt nichtparametrischer Flaschen, Annali di Mat. Pura et App1. 4 (44), 1957, 14-133.

3. Kurzweil, J. Nichabsolut kovergente Integrale, Teubner Texte zur Mathematik, No. 26, Leipzig, 1980 .

4. Kurzweil, J. On multiplication of Perron-integrable functions, Czech. Math J. 23 (98), (1973), 542-566. 
5. Namioka, I. and Kel1y, J., Linear topologlcal spaces, D. Van Nostrand, Princeton, 1963.

6. Ostaszewski, K. A topology for the spaces of Denjoy-integrable functions, Proceedings of the Sixth Summer Real Analysis Symposium, Real Analysis Exchange 9 (1), (1983-84), 79-85.

7. Ostaszewski, K. Henstock Integration in the P1ane, Memoirs Amer. Math. Soc., 353, September 1986.

8. Pfeffer, W.F. The divergence theorem, Transactions of the Amer. Math. Soc. 295 (2), $1986,665-685$.

9. Thomson, B.S. Spaces of conditionally integrable functions, J. London Math. Soc. (2), $2(1070), 358-360$. 


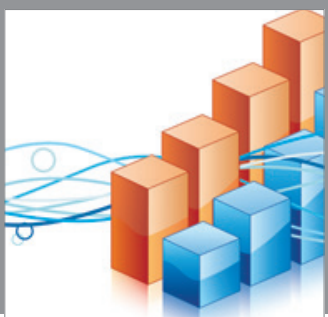

Advances in

Operations Research

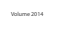

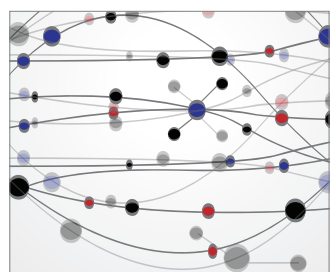

\section{The Scientific} World Journal
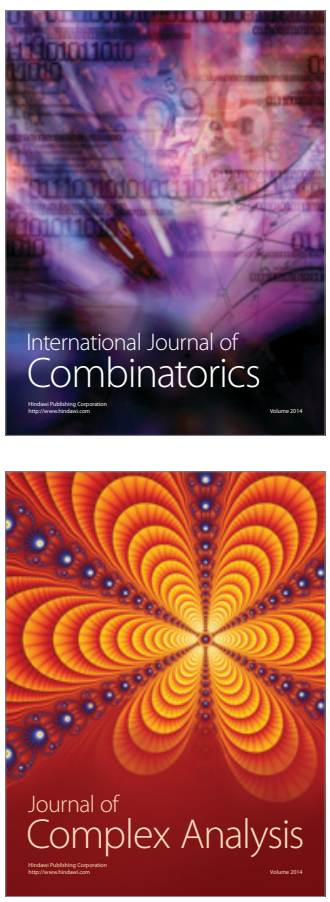

International Journal of

Mathematics and

Mathematical

Sciences
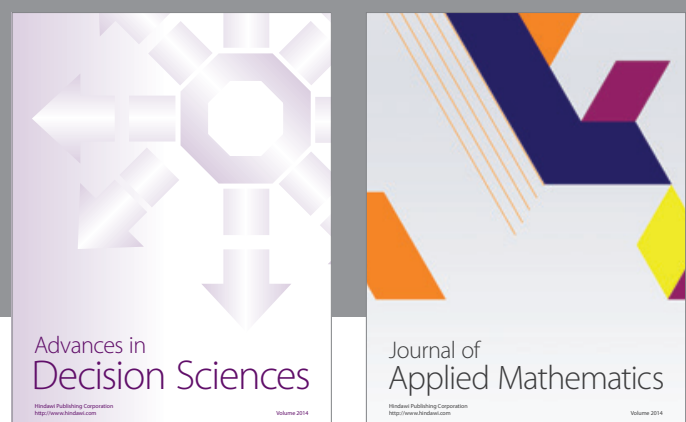

Journal of

Applied Mathematics
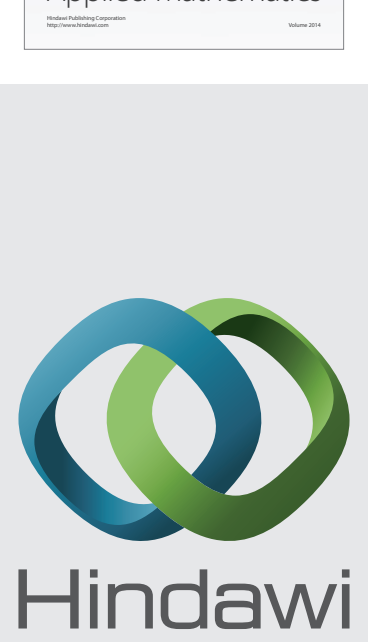

Submit your manuscripts at http://www.hindawi.com
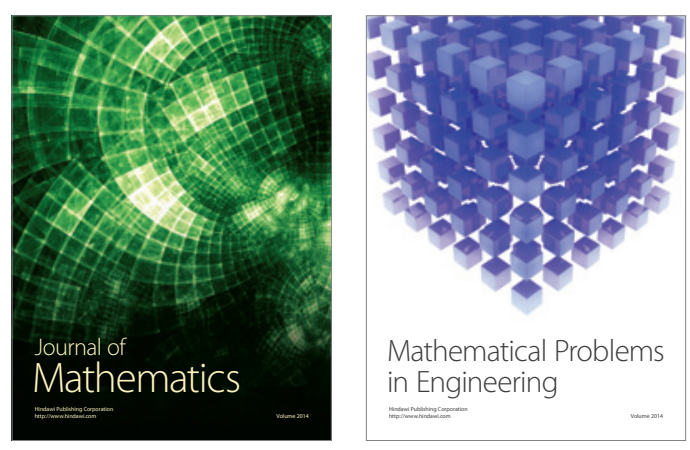

Mathematical Problems in Engineering
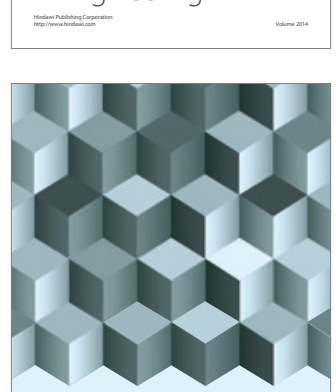

Journal of

Function Spaces
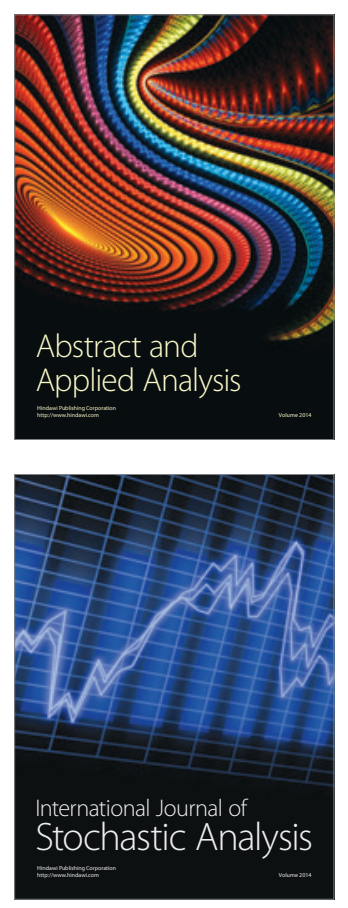

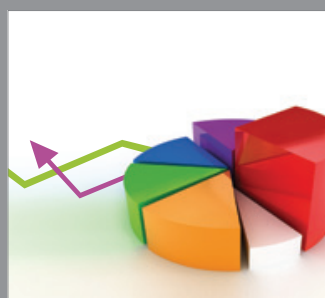

ournal of

Probability and Statistics

Promensencen
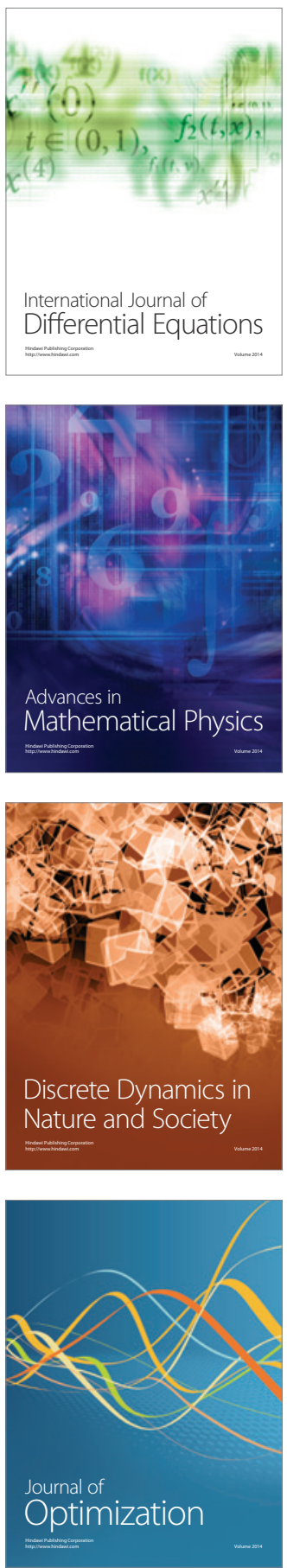